\title{
Relevant social and spatial contexts for elementary school children: An examination of multiple scales
}

\begin{abstract}
This study examined direct area-level effects of 4 common child well-being outcomes across multiple scales of residential and school neighborhoods to identify relevant contexts for measuring neighborhood effects on elementary school children. Results from growth curve models indicate that neighborhoods operationalized as residential and school census tracts exerted similar effect sizes while neighborhoods operationalized as school attendance zones showed attenuated effects. These results suggest that it may be reasonable to interchange residential and school census tracts when examining contextual effects on child well-being. In addition, results suggest that school attendance zones represent conservative, theoretically sound neighborhoods for elementary school children.
\end{abstract}

\section{KEYWORDS:}

Neighborhood; Longitudinal; School Attendance Zone; MAUP; School Neighborhood

\section{RESEARCH HIGHLIGHTS}

- Direct effects of residential and school neighborhoods were examined on 4 common child well-being outcomes

- Residential and school census tracts may be interchanged in light of data limitations

- School attendance zones may be a proxy for children's activity spaces

- School attendance zones represent a conservative, theoretically sound neighborhood for children 


\section{INTRODUCTION}

A recurring issue in the neighborhood effects literature is the definition and operationalization of a "neighborhood" or relevant geographic areas. Neighborhoods can be defined in many ways, but researchers agree that the definition and scale of a neighborhood should be based on theory and evidence specific to the outcome(s) under study and the hypothesized pathways through which neighborhoods exert influence (Diez Roux \& Mair, 2010; Flowerdew, Manley, \& Sabel, 2008; Root, 2012; Sharkey \& Faber, 2014). However, neighborhood analyses often takes place in the presence of data constraints and as a result, researchers often use administrative units such as census tracts to define neighborhood boundaries. Yet, the use of administrative boundaries may not be arbitrary for children as they have limited mobility and independence, and are often constrained to the spaces surrounding them (Leventhal \& Brooks-Gunn, 2003; Northridge, Sclar, \& Biswas, 2003; Sharkey \& Faber, 2014).

The residential census tract is the most frequently used definition of "neighborhood" in neighborhood effect studies during childhood. Considered as a whole, the body of research conducted at the level of the residential census tract demonstrates that characteristics of the neighborhood's population, e.g., poverty, appear to be consistently linked with children's academic or developmental trajectories (Leventhal \& Brooks-Gunn, 2000; Sastry \& Pebley, 2010; Sharkey \& Faber, 2014). Yet, residential neighborhoods are only a subset of the potentially relevant contextual influences on children. As such, recent research urges neighborhood and health scholars to define neighborhoods as contexts that are relevant to the social and spatial environments in which children regularly engage (Sharkey \& Faber, 2014). Given that youth spend a large portion of their day in school, school neighborhoods serve as a natural starting point for redefining relevant contexts. Yet, the role of school neighborhoods have 
largely been ignored in this literature except for a few city-specific studies that have examined the effects of school neighborhoods on youth health and well-being using school attendance boundaries, cluster analysis, school census tracts, and school-centric buffers (Bernelius \& Kauppinen, 2012; Forsyth, Wall, Larson, Story, \& Neumark-Sztainer, 2012; Schwartz, 2010; Whipple, Evans, Barry, \& Maxwell, 2010; Zhang, Christoffel, Mason, \& Liu, 2006).

In light of children's limited mobility and daily exposure to both home and school neighborhood environments, school census tracts and school attendance zones may represent valid contexts that exert influence on health, well-being, and development during elementary school. However, which "scale" is most appropriate for defining relevant social and spatial contexts? That is, is a school attendance zone a more salient neighborhood than that of the census tract where the school is located, or vice-versa? Using simulated cities, Spielman, Yoo, and Linkletter (2013) found that neighborhood effects were strongly influenced by the definition of neighborhood - neighborhoods that were "too small" overestimated neighborhood effects on behaviors whereas neighborhoods that were "too big" underestimated neighborhood effects. Thus, researchers often conduct parallel analyses at different scales and compare neighborhood effect estimates (Flowerdew et al., 2008; Galster, 2008; Root, 2012). The challenge, then, is to find a balance between the modifiable areal unit problem (correlations between outcomes and context are often stronger at smaller spatial scales) or spatial diffusion (characteristics tend to cluster spatially and become more heterogeneous in larger areas) and theoretical rationale about why and how neighborhoods exert influence within each scale.

Neighborhoods provide the physical spaces in which youth access resources and opportunities, but also the social spaces in which interactions with peers, family, and other adults occur (Leventhal et al., 2009). As such, researchers have identified several broad underlying 
mechanisms - institutional resources, collective socialization, collective efficacy, social capital, and social organization - through which neighborhoods may influence health, well-being, and development (Jencks \& Mayer, 1990; Leventhal \& Brooks-Gunn, 2000; Sampson et al., 2002). Although each theoretical perspective conceptualizes the mechanisms differently, each argues that neighborhoods are both a physical and social space in which interactions with parents, other adults (e.g., teachers, coaches, librarians, neighbors), and peers foster healthy development. As such, the definition and scale of neighborhood should be based on the underlying theoretical mechanisms linking exposure to outcome. For instance, mechanisms that focus on institutions, such as the research on school quality, suggest the context most salient for analysis may be the school attendance zone where resources, e.g., the tax base related to school funding, are drawn from (Sharkey \& Faber, 2014).

The present paper examines the relationship between residential and school neighborhoods and four commonly used child well-being outcomes (educational achievement and psychosocial adjustment [see Curtis et al., 2013 and Sharkey \& Faber, 2014]) in U.S. elementary school children. The intent of this paper is not to delve into the mechanisms underlying the association between neighborhood and child well-being but rather to examine how those associations vary across contexts that are relevant to school-aged children. Specifically, I examine the direct area-level effects on reading and math scores and internalizing and externalizing behaviors across neighborhoods defined by residential census tracts, school census tracts, and school attendance zones characterized by commonly used sociodemographic features. Because school attendance zones encompass both residential and school tracts, I hypothesize that the "neighborhood effects" will be attenuated relative to tract neighborhoods, which are smaller in scale. I also expect to find similar effect sizes across residential and school 
census tracts because elementary schools draw from the immediate surrounding areas (Whipple et al., 2010), making it likely that residential and school tracts share similar sociodemographic characteristics. To date, no research has used nationally representative data to demonstrate how school neighborhoods are associated with educational achievement and psychosocial adjustment during elementary school.

\section{METHODS}

\section{Data}

The Early Childhood Longitudinal Study - Kindergarten Cohort (ECLS-K), sponsored by the Department of Education (National Center for Education Statistics, 2006), is a nationally representative study that followed a cohort of more than 21,400 children who entered kindergarten during the 1998-1999 school year through $8^{\text {th }}$ grade. Data collection took place during the fall and spring of kindergarten (1998-1999) and $1^{\text {st }}$ grade (1999-2000) and the spring of $3^{\text {rd }}(2002), 5^{\text {th }}(2004)$, and $8^{\text {th }}(2007)$ grades. The ECLS-K employed a multistage probability sample design (National Center for Education Statistics, 2006). In the base year the primary sampling units (PSUs) were geographic areas consisting of counties or groups of counties. From these PSUs, approximately 24 children were randomly sampled from each of the 1,277 selected schools, both public and private. In order to maximize the amount of longitudinal data, subsamples of children were followed if they changed schools and any child flagged to be followed at one point in time continued to be followed in subsequent data collections. In a longitudinal sample, attrition due to non-response and eligibility change is expected. During the first four waves (kindergarten through $5^{\text {th }}$ grade), the ECLS-K had a $40 \%$ attrition rate (National Center for Education Statistics, 2006). However, the ECLS-K included weights to compensate 
for both sampling strategy and attrition; all analyses in this study are weighted appropriately. Results of weighted analyses are generalizable to the U.S. population of kindergarten children in the 1998-1999 school year and first graders in 1999-2000. Subsequent waves are only representative of the ECLS-K cohort (National Center for Education Statistics, 2006).

The ECLS-K contains longitudinal and geocoded data collected directly from children, parents, teachers, and school administrators, providing comprehensive information on education, development, and home, school, and neighborhood environments. The ECLS-K did not provide census tract geocodes for the $5^{\text {th }}$ grade; thus, $5^{\text {th }}$ grade geographic information was only available for children who did not move in elementary school ( $83 \%$ of the sample). Kindergarten, $1^{\text {st }}$, and $3^{\text {rd }}$ grade geocodes were linked to the 2000 US Decennial Census; $5^{\text {th }}$ grade geocodes were linked to the 2005-2009 American Community Survey (ACS) 5-year estimates.

\section{Outcomes}

Academic achievement from kindergarten to $5^{\text {th }}$ grade was measured using reading $(\mathrm{N}=9,790)$ and math $(\mathrm{N}=9,810)$ scores calculated from item response theory (IRT) procedures. "IRT uses the pattern of right, wrong, and omitted responses to the items actually administered in an assessment and the difficulty, discriminating ability, and 'guess-ability' of each item to place each child on a continuous ability scale" (National Center for Education Statistics, 2006, p. 3-5). Responses across waves were pooled to stabilize longitudinal estimates; the child's response at each wave represents estimates of the number of items the child would have answered correctly at each point in time if they had taken all of the 186 questions in the reading forms and all of the 153 questions in all of the mathematics forms (National Center for Education Statistics, 2006). Scores were standardized with a mean of 50 and a standard deviation of 10 (Table 1). 
Internalizing $(\mathrm{N}=16,080)$ and externalizing $(\mathrm{N}=16,160)$ behaviors were measured using subscales of the Teacher Social Rating Scale for fall and spring kindergarten and spring of $1^{\text {st }}$, $3^{\text {rd }}$, and $5^{\text {th }}$ grades. The five to six items of the Externalizing Problem Behaviors scale measured acting out behaviors (e.g., arguing, fighting, showing anger, acting impulsively, disturbing the classroom's ongoing activities). The four items on the Internalizing Problem Behavior measured negative affective states such as anxiety, loneliness, sadness and low self-esteem. The ECLS-K scored each scale as the average of the underlying items. The National Center for Educational Statistics (2006) reports adequate split-half reliability ranges of 0.76 to 0.89 for the Externalizing and Internalizing Problem Behavior scales. Exploratory and confirmatory factor analyses confirmed the full scale's structure. Scale scores were standardized with mean of 0 and standard deviation of 1 (Table 1).

\section{Child-, Family-, and School-level Controls}

I controlled for child and family time-invariant measures including child race/ethnicity and child sex, as well as time-varying controls including child health status, child age, family structure, maternal education, socioeconomic status (SES), residential mobility, and direct and indirect measures of parental involvement in the child's education. School context was measured by time-varying principal report of the percentage of minority students, whether parents were active in school programs, and whether there was a problem with teacher turnover (Table 1). 
Table 1. Descriptive statistics of child, family, and school, characteristics for reading and math achievement and internalizing and externalizing behaviors. Weighted and pooled sample from the ECLS-K sample - kindergarten through $5^{\text {th }}$ grade.

\begin{tabular}{|c|c|c|c|c|c|}
\hline & \multicolumn{5}{|c|}{ Pooled Sample } \\
\hline & $\mathbf{N}$ & Mean & SE & Min & Max \\
\hline \multicolumn{6}{|l|}{ Outcomes } \\
\hline Reading Scores & 40700 & 49.79 & 0.24 & 15.00 & 143.68 \\
\hline Math Scores & 41580 & 49.84 & 0.27 & 18.62 & 120.17 \\
\hline Internalizing Behaviors & 52130 & 0.01 & 0.01 & -1.17 & 4.67 \\
\hline Externalizing Behaviors & 52480 & 0.02 & 0.01 & -1.14 & 4.06 \\
\hline \multicolumn{6}{|l|}{ Child and Family Characteristics } \\
\hline \multicolumn{6}{|l|}{ Race and Ethnicity } \\
\hline White & 60110 & 0.58 & 0.02 & 0 & 1 \\
\hline Black & 60110 & 0.15 & 0.02 & 0 & 1 \\
\hline Hispanic & 60110 & 0.19 & 0.02 & 0 & 1 \\
\hline Other & 60110 & 0.07 & 0.01 & 0 & 1 \\
\hline Child in good health & 60110 & 0.83 & 0.01 & 0 & 1 \\
\hline Male & 60300 & 0.52 & 0.01 & 0 & 1 \\
\hline Parents married & 57030 & 0.71 & 0.01 & 0 & 1 \\
\hline Residential Mobility & 41350 & 0.14 & 0.00 & 0 & 1 \\
\hline \multicolumn{6}{|l|}{ Maternal Education } \\
\hline LTHS & 53410 & 0.13 & 0.01 & 0 & 1 \\
\hline High school and some college & 53410 & 0.63 & 0.01 & 0 & 1 \\
\hline Bachelor's degree & 53410 & 0.18 & 0.01 & 0 & 1 \\
\hline Graduate or professional degree & 53410 & 0.07 & 0.00 & 0 & 1 \\
\hline SES & 57440 & -0.03 & 0.03 & -4.75 & 2.88 \\
\hline Parent Communication & 56720 & 2.61 & 0.05 & 0 & 99 \\
\hline Parental Involvement in Education & 57100 & -0.01 & 0.02 & -3.89 & 2.18 \\
\hline \multicolumn{6}{|l|}{ School Characteristics } \\
\hline \multicolumn{6}{|l|}{$\%$ Minority Students } \\
\hline $0-<25 \%$ & 57660 & 0.49 & 0.03 & 0 & 1 \\
\hline $25-<50 \%$ & 57660 & 0.17 & 0.01 & 0 & 1 \\
\hline $50-<75 \%$ & 57660 & 0.12 & 0.01 & 0 & 1 \\
\hline$>=75 \%$ & 57660 & 0.23 & 0.02 & 0 & 1 \\
\hline Parents are active in school programs & 46460 & 0.78 & 0.01 & 0 & 1 \\
\hline Problem with teacher turnover & 46490 & 0.07 & 0.01 & 0 & 1 \\
\hline
\end{tabular}

$\mathrm{N}=9,810$ students for math achievement; $\mathrm{N}=9,790$ students for reading achievement. Reading and math scores were standardized with mean $=50$ and standard deviation $=10 . \mathrm{N}=16,080$ students for internalizing behaviors; 16,160 students for externalizing behaviors. Internalizing and externalizing behavior scores were standardized with mean=1 and standard deviation $=0$. Per ECLS-K guidelines, all N's are rounded to the nearest 10 to protect student privacy and weighted with sample weights. 
The SES measure used in this study is a continuous scale, constructed by the ECLS-K at each wave, combining information on household-level education, occupation, and income. Parent Communication was measured by asking parents "how many of your child's classmates' parents do you speak with regularly?" Parental involvement in education was created by summing positive responses on questions such as "attended an open house," "met the child's teacher," or "talk with child about their plan after graduating high school." Because the number of questions varied at each survey wave, the Parent Involvement in Education score was standardized to make the scale comparable across waves. Residential mobility was defined as a time-varying binary indicator of whether or not the child moved in each wave. 
Neighborhood Construction and Characterization - Residential Tracts, School Tracts, School Attendance Zones

Time-varying neighborhood contexts were defined using residential census tracts, school census tracts, and school attendance zones. Census tract neighborhoods were defined using the tract in which the child lived and the tract in which the school was located. Official school attendance zones (SAZs) were not available on a national scale for the years this study took place.

Therefore, I created SAZs by aggregating the school tract and each residential tract associated with that school.

Residential tracts, school tracts, and SAZs were characterized using four time-varying sociodemographic attributes commonly found in neighborhood effect studies during childhood (Leventhal \& Brooks-Gunn, 2000; Sampson, Morenoff, \& Gannon-Rowley, 2002): proportion of residents living below the federal poverty line, proportion of residents with less than a high school education, and proportion of affluent residents - those with at least 4-year degrees. I then transformed the raw proportions into binary values by cutting the variable above or below the median (Table 2). I transformed the neighborhood variables to support propensity score methodology using binary treatments (Rosenbaum \& Rubin, 1983). 
Table 2. Descriptive Analysis of Neighborhood Contexts: Residential Tracts, School Tracts, School Attendance Zones

\begin{tabular}{llllll}
\hline & \multicolumn{6}{c}{$\begin{array}{c}\text { Residential Tracts, School Tracts, } \\
\text { School Attendance Zones }\end{array}$} \\
\cline { 2 - 6 } & Median & Mean & SD & Min & Max \\
\cline { 2 - 6 } & & & & & \\
Residential Tracts & 0.09 & 0.13 & 0.11 & 0.00 & 0.77 \\
$\quad$ Poverty & 0.16 & 0.20 & 0.15 & 0.00 & 0.86 \\
$\quad$ Less than High School Education & 0.19 & 0.24 & 0.17 & 0.00 & 0.90 \\
$\quad$ Affluence & & & & & \\
$\quad$ School Tracts & 0.10 & 0.14 & 0.12 & 0.00 & 0.77 \\
$\quad$ Poverty & 0.17 & 0.20 & 0.15 & 0.00 & 0.82 \\
$\quad$ Less than High School Education & 0.19 & 0.24 & 0.17 & 0.00 & 0.88 \\
$\quad$ Affluence & & & & & \\
School Attendance Zones & 0.10 & 0.13 & 0.10 & 0.00 & 0.77 \\
$\quad$ Poverty & 0.18 & 0.20 & 0.13 & 0.00 & 0.82 \\
$\quad$ Less than High School Education & 0.20 & 0.24 & 0.15 & 0.00 & 0.88 \\
$\quad$ Affluence & & & & & \\
\hline
\end{tabular}




\section{Analytic Strategy}

Time-varying Propensity Scores (TVPS)

Because children moved over the course of the survey, time-varying propensity scores are used to mitigate selection bias at each wave (Reference Removed for Review; Reference Removed for Review). For census tract and school attendance zone neighborhoods I used logistic regression models to create propensity scores for each child for each characterization of neighborhood at each wave of the survey. In each model, I regressed neighborhood type (reference set as "low") on family characteristics from the same wave; besides child race/ethnicity and residential mobility, background characteristics included in the propensity score equations were not duplicated in growth curve models (see supplemental materials for a list of variables). The predicted probability from each model represents the propensity score, or the likelihood that a family selected into their neighborhood type based on background characteristics (Rosenbaum \& Rubin, 1983).

\section{Multilevel Growth Curve Models}

The multivariate analyses use growth curve models for continuous outcomes to predict trajectories of reading and math achievement and internalizing and externalizing behaviors by analyzing time points (Level 1) nested within individual children (Level 2). Thus, the child's grade is the Level 1 unit, and child is Level 2 (Singer \& Willett, 2003). Because there were not enough students per residential census tract to estimate 3-level models, I did not estimate 3-level models for any of the school tracts or school attendance zones for the sake of comparability.

I first compared linear and quadratic functions of grade at Level 1. For math scores, as well as internalizing and externalizing behaviors, the linear model was the best fit, suggesting 
that scores changed uniformly over time. For reading scores, the quadratic model was the best fit, suggesting that scores increased as children progressed but eventually leveled off. Models were estimated using xtmixed in Stata 12 (StataCorp, 2011) with child-, family-, and school-level controls, time-varying propensity scores to adjust for selection, as well as probability weights and a sandwich estimator of standard errors, which adjusted for clustering within the primary sampling unit. I assigned one unique variance parameter per random effect and assumed the covariance parameters were zero because of insufficient evidence to estimate individual slopes. The basic model specification is:

$$
\begin{gathered}
Y_{i j}=\pi_{0 i}+\pi_{1 i} G R A D E_{i j}+\pi_{2 n i} W_{i}+\varepsilon_{i j} \\
\text { where: } \pi_{0 i}=\gamma_{00}+\gamma_{0 n} X_{i}+\xi_{0 i} \\
\pi_{1 i}=\gamma_{10}+\gamma_{1 n} Z_{i}+\xi_{1 i}
\end{gathered}
$$

$Y_{i j}$ shows the level-1 model which includes GRADE (centered at kindergarten) as the trajectory of educational or psychosocial outcomes across students and $W$ as a matrix of time-varying predictors of educational achievement. $\pi_{0 i}$ and $\pi_{0 i}$ are the level- 2 models which show how the initial status and trajectory are modified over time. $X_{i}$ and $Z_{i}$ are matrices of time-invariant variables that modify the intercept and slope over time, respectively. I use the Bayesian information criterion (BIC) and the Akaike information criteria (AIC) to assess model fit.

\section{RESULTS}

\section{Descriptive Analysis}

Table 1 summarizes the descriptive findings for child, family, and school characteristics. Approximately $58 \%$ of the sample self-identified as non-Hispanic white, $0.15 \%$ non-Hispanic black, 19\% Hispanic, and 7\% non-Hispanic Asian or other racial backgrounds. On average, 
children were in good health $(\mathrm{M}=0.83 ; \mathrm{SE}=0.01), 71 \%$ lived in a household with married parents ( $\mathrm{SE}=0.01), 63 \%$ of mothers had a high school diploma with some college experience $(\mathrm{SE}=0.01)$, and $14 \%$ of children moved during elementary school $(\mathrm{SE}=0.00)$. Of the schools students attended, approximately half were comprised of less than $25 \%$ minority students $(\mathrm{M}=0.491 \mathrm{SE}=0.03)$, while approximately one-fifth were composed of $75 \%$ or more minority students $(\mathrm{SE}=0.02)$. Table 2 presents a descriptive analysis of neighborhoods defined by residential census tract, school census tracts, and school attendance zones (SAZ). Sociodemographic characteristics were similar across types of neighborhoods. On average, students were exposed to neighborhoods in which $13 \%$ of residents lived below the federal poverty line, $20 \%$ of residents obtained less than a high school diploma, and $24 \%$ of residents had a graduate or professional degree.

\section{Growth Curve Models}

Table 3 presents coefficients from the final growth curve models estimating the direct association between neighborhood context and educational achievement and psychosocial adjustment during elementary school; these models included child-, family-, and school-level controls as well as time varying propensity scores to adjust for selection bias. Due to convergence or over-fitting issues, I was unable to interact grade by neighborhood to examine neighborhood effects on educational or psychosocial trajectories. Thus, coefficients represent neighborhood effects during kindergarten. Overall, residential tracts, school tracts, and SAZs showed similar associations with initial reading and math scores while all neighborhood contexts showed little or no association with either internalizing or externalizing behaviors. 
Residential census tracts characterized by high poverty, low levels of education, and high affluence were significantly associated with reading and math achievement scores. Absolute effect sizes for differences in the initial status of reading and math scores ranged from 0.33 to 0.96 standard deviations (Table 3), with affluent neighborhoods exerting the strongest effect. School tracts characterized by high poverty, low levels of education, and high affluence were associated with medium to large absolute effect sizes on reading and math achievement, 0.51 to 0.85 standard deviations. School attendance zones showed similar, but attenuated effects for reading achievement (absolute effect sizes range from 0.25 to 0.70 ), and only SAZs characterized by poverty and affluence were significantly associated with math scores. 
Table 3. Coefficients from final growth curve models estimating neighborhood effects on educational achievement and psychosocial adjustment from kindergarten to $5^{\text {th }}$ grade, net of family characteristics, school context, and time-varying propensity scores.

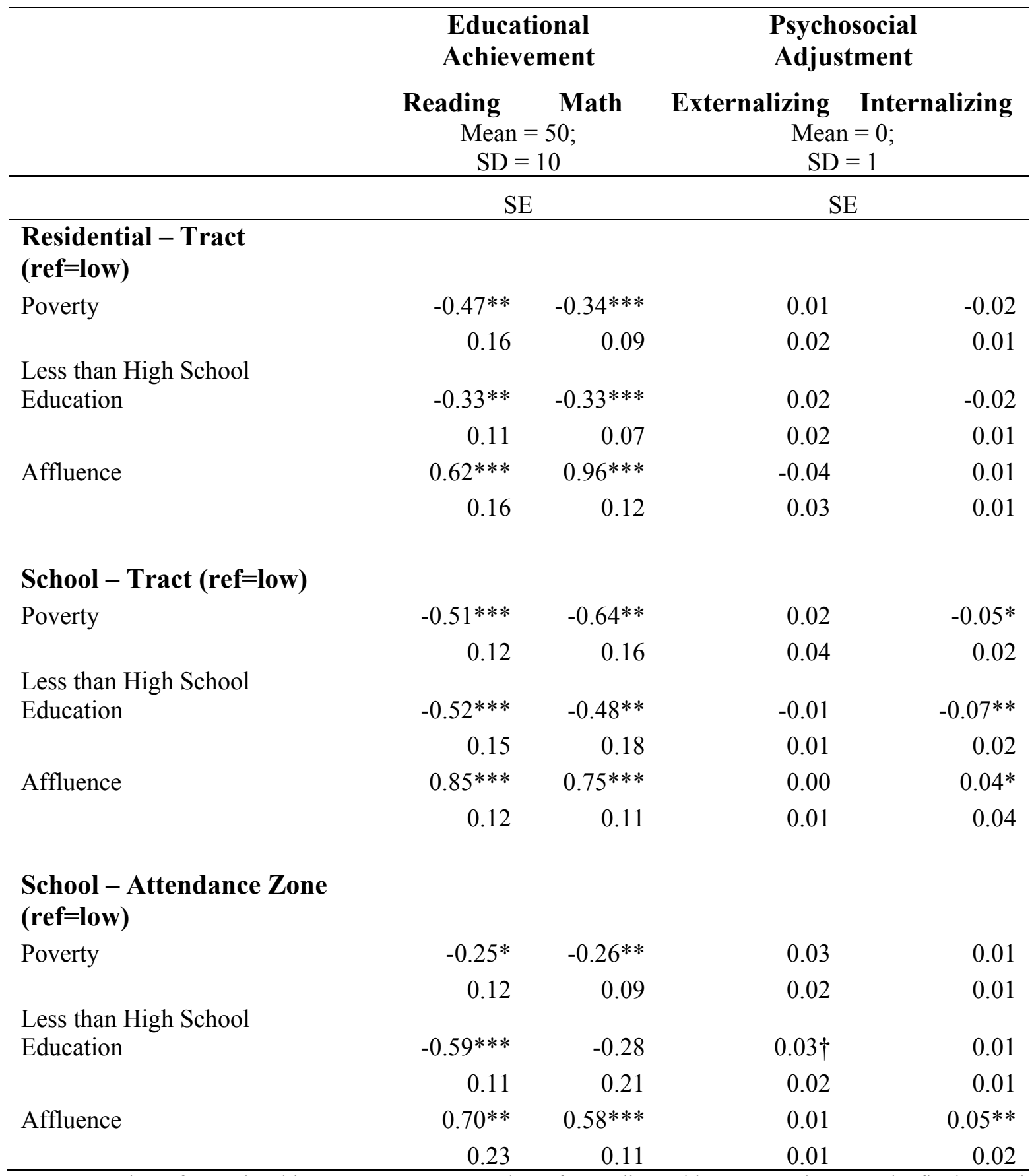

$\mathrm{N}=9,810$ students for math achievement; $\mathrm{N}=9,790$ students for reading achievement. These are the final growth curve models, which include controls for: child's race/ethnicity, health status, sex, parental marital status, maternal education, family SES, parent communication with other parents, level of involvement with child's education, residential mobility, school minority levels, culture of active parenting within schools, problems with teacher turnover in schools, and time-varying propensity scores to mitigate selection bias. Each model is estimated with probability weights and sandwich estimators. 
${ }^{b} \mathrm{~N}=16,080$ students for internalizing behaviors; 16,160 students for externalizing behaviors These are the final growth curve models, which include controls for: child's race/ethnicity, health status, sex, age, parental marital status, maternal education, family SES, residential mobility, school minority levels, culture of active parenting within schools, and time-varying propensity scores to mitigate selection bias. Each model is estimated with probability weights and sandwich estimators.

† $p<0.10 .{ }^{*} p<0.05$. ${ }^{* *} p<0.01 . * * * p<0.001$. 


\section{DISCUSSION}

Using nationally representative data that followed U.S. children from kindergarten to $5^{\text {th }}$ grade, this study examined area-level effects on reading and math achievement and internalizing and externalizing behaviors across neighborhoods defined by residential census tracts, school census tracts, and school attendance zones. Moreover, within the neighborhood definitions, I examined effects across a number of sociodemographic characterizations previously linked to childhood educational and behavioral outcomes. I hypothesized that the effect sizes associated with residential and school census tracts would be similar to each other and larger than the effect sizes associated with school attendance zone neighborhoods. Results from growth curve models generally supported these hypotheses. Since time-varying propensity scores were used to mitigate selection into neighborhoods, I do not believe these effects are due to selection bias.

As expected, residential census tracts were significantly associated with reading and math scores, but not internalizing and externalizing behaviors. For instance, children living in affluent tracts scored 0.96 standard deviations higher on kindergarten math scores relative to children living in tracts with low affluence. This effect translates into an almost 10 point difference in math scores for kindergarten children. Ample evidence suggests early disparities generally do not converge, but rather widen leading to greater educational disparities in adolescence and adulthood (Entwisle, Alexander, \& Olson, 2005), which speaks directly to the importance and impact of social and environmental contexts. Similarly, school census tracts supported my hypothesis and demonstrated comparable patterns and effects sizes. The similarity between residential and school tracts may be due to the fact that $32 \%$ of kindergartners lived in the same tract as the school they attended. A correlation analysis (not shown) confirmed a correlation of $0.99(p<0.001)$ between school and residential neighborhood sociodemographic contexts. 
Further, elementary schools are often "neighborhood" schools that draw from the immediate surrounding areas (Whipple et al., 2010). In light of this, I believe residential and school census tracts, while conceptually different, may actually be so similar that it is reasonable to interchange them when examining contextual influences on health, development, and well-being of elementary school children.

One of the major criticisms of neighborhood effects research is the lack of theoretical mechanisms linking neighborhood context to child well-being outcomes (Leventhal \& BrooksGunn, 2000; Sampson et al., 2002; Sharkey \& Faber, 2014). That is, "how" do neighborhoods exert influence? There has been significant theoretical and empirical progress in this area, particularly with the use of structural equation modeling, parallel multiple mediator models, and analysis of longitudinal data (Dupere, Leventhal, Crosnoe, \& Dion, 2010; Jencks \& Mayer, 1990; Kohen, Dahinten, Leventhal, \& McIntosh, 2008; Leventhal \& Brooks-Gunn, 2000). Part of the challenge of this area of research is the use of the term "neighborhood." This term muddies the water, so to speak, by carrying connotations of a sense of community, indicates that interactions with neighbors regularly occur and influence well-being, and have clearly defined physical and social boundaries (Diez Roux \& Mair, 2010; Sharkey \& Faber, 2014). I think, for children this age (kindergarten through $5^{\text {th }}$ grade), who have limited autonomy, the area around the home or school in which they regularly engage represents a theoretically strong environment for capturing how context impacts educational and developmental outcomes. For instance, schools are, in large part, funded by the local tax revenues based on local property values and business activities. The local financial capital generates better infrastructure, which translates into human capital. Thus, schools in advantaged areas are often able to hire and retain highly qualified, effective teachers, and create a culture where parents expect and lobby for high quality 
services and become involved with school activities such as parent-teacher organizations (Crosnoe, 2009; Dupere et al., 2010; Lareau, 2003; Leventhal, Dupere, \& Brooks-Gunn, 2009; Owens, 2010). Thus, the structural factors from the surrounding areas create a "safety net" that promotes educational achievement and supports children's socioemotional development (Roeser \& Eccles, 2014).

In addition to residential and school census tracts, I examined the association between school attendance zones and educational and behavioral outcomes during elementary school. I hypothesized that effects for school attendance zones would be attenuated relative to census tract neighborhoods, which my results support. I argue that elementary SAZs represent the ideal local context for children as they: (1) simultaneously represent the area around the home and the school that children are exposed to each day, (2) are often larger than a census tract, but not so big that they overshadow the limited mobility of children this age, and (3) may provide a more accurate depiction of children's exposure to or interaction with their environments than traditional contexts defined via administrative boundaries (Dobbie \& Fryer, 2011; Jones \& Pebley, 2014; Schwartz, 2010; Sharkey \& Faber, 2014; Villaneuva et al., 2012). If census tracts are good proxies for "neighborhoods" then we would expect to find that effects for SAZs are slightly attenuated because of processes of diffusion. Areas of similar sociodemographic characteristics tend to cluster together spatially vis-a-vie Tobler's first law of geography, "everything is related to everything else, but near things are more related than distant things" (Tobler, 1970, p. 236). Thus, within SAZs, the further away you move from the core area around the home or school, contextual environments should become more heterogeneous and exert less influence over child development (Crowder \& South, 2011; Downey, 2006). My study found this to be true, and theoretically and empirically support the use of school attendance zones as 
important environments to consider for impacting child development, particularly educational achievement.

Theoretically, school attendance zones may also represent children's activity spaces geographic units which combine the child's home area as well as other places he or she routinely visits (Jones \& Pebley, 2014; Villaneuva et al., 2012). Because SAZs are comprised of the area around the child's home, the area around the child's school, and all of the tracts that feed into that school, I argue that SAZs are a good proxy for activity spaces because they are able to capture area-level exposures not just of home and school environments, but also other local areas, such as a friend's home. Activity spaces for children have received very little attention since the 1980's, partially due to the strict privacy regulations for children, but are resurging due to the theoretical utility and advancements in geographic technology. However, creating activity spaces are methodologically time-consuming and often require subjects to wear GPS units, keep travel diaries, or both. In light of these data limitations, school attendance zones represent a plausible and time-efficient alternative to activity spaces. Previously, official school attendance zones were often unavailable to researchers without considerable time and effort in obtaining them. The National Historic Geographic Information System (NHGIS) now hosts national school catchment areas available through the School Attendance Boundary Information System (SABINS) (The College of William and Mary and the Minnesota Population Center, 2011) for the 2009, 2010, and 2011 academic school years. The National Center for Education Statistics hosts boundary files for the 2013 academic year via the School Attendance Boundary Surveys (Phan, 2015). These boundary files line up with the new and geocoded kindergarten, $1^{\text {st }}$, and $2^{\text {nd }}$ grade waves of the ECLS-K:2011 surveys. 
There is no single correct scale to measure "neighborhood", but scale affects both the inferences we make about neighborhood effects as well as the theoretical underpinnings linking context to outcome (Galster, 2008). Flowerdew and Colleagues (2008) compared existing neighborhood boundaries to five realistically defined pseudo areas, and found that results vary depending on "where you draw the lines." They argue that researchers need to think of “effective" neighborhoods and experiment with different scales and aggregations. Both census tracts and school attendance zones may represent "effective" neighborhoods for children. Yet, census tracts may represent neighborhoods that are "too small" and lead to overestimation of neighborhood effects. On the other hand, school attendance zones may be "too big" and lead to underestimation of neighborhood effects (Spielman et al., 2013). As such, I believe school attendance zones yield more conservative estimates of neighborhood effects and represent theoretically sound neighborhoods for children.

\section{LIMITATIONS}

This study has several limitations. First, I estimated elementary school attendance boundaries using census tracts for each school and sampled child rather than official catchment areas. Thus, it is possible that I underestimated the size of SAZs. However, each school sampled roughly 24 children and catchment areas for elementary schools are generally small and confined to the surrounding areas (Whipple et al., 2010). Moreover, I created SAZs that are time-varying in order to keep up with constantly change official attendance boundaries (Coulton \& Korbin, 2007). Second, residential tracts, school tracts, and school attendance zones were characterized in crude ways via low/high sociodemographic characteristics, which may mask significant variation. However, identifying more nuanced thresholds is difficult at a national scale, and I 
believe this research demonstrates the validity of contextual boundaries rather than the cutoffs used to characterize them. Third, the reading and math outcomes used in this study were scaled to the $5^{\text {th }}$ grade. Thus, these measures were only available for children who remained in the survey through elementary school. However, I limited bias as much as possible by estimating models with survey weights that compensated for attrition. Another limitation is that the results are only generalizable to the U.S. population of kindergarten children in the 1998-1999 school year and first graders in 1999-2000; subsequent waves are only representative of the ECLS-K cohort. While this is important to consider when interpreting results, I also point out that the ECLS-K surveyed a large and diverse population of children, which is a strength of the dataset and my analyses.

\section{CONCLUSION}

Despite these limitations, my findings have important implications for future neighborhood research and policy and speak directly to the importance and impact of social and environmental contexts. This paper contributes to the growing literature on contextual influences on childhood health, well-being, and development during a critical stage of the life course. By using an inclusive growth curve modeling approach that simultaneously controlled for time-varying family and child characteristics, school context, and propensity scores to mitigate selection bias, I demonstrated the utility of residential census tracts, school census tracts, and school attendance zones as neighborhood proxies for elementary school children. In particular, this research contributed to the growing literature identifying an appropriate scale for evaluating neighborhood impacts on education during childhood. 


\section{REFERENCES}

Bernelius, V., \& Kauppinen, T. M. (2012). School Outcomes and Neighbourhood Effects: A New Approach Using Data from Finland. In M. van Ham, D. Manley, N. Bailey, L. Simpson \& D. Maclennan (Eds.), Neighbourhoood Effects Research: New Perspectives (pp. 225-248). Dordrecht: Springer Science+Business Media.

Coulton, C., \& Korbin, J. (2007). Indicators of child well-being through a neighborhood lens. Social Indictors Research, 84(3), 349-361.

Crosnoe, R. (2009). Low-Income Students and the Socioeconomic Composition of Public High Schools. American Sociological Review, 74(5), 709-730.

Crowder, K., \& South, S. J. (2011). Spatial and temporal dimensions of neighborhood effects on high school graduation. Social Science Research, 40(1), 87-106.

Curtis, S., Pain, R., Fuller, S., Khatib, Y., Rothon, C., Stansfeld, S., et al. (2013). Neighbourhood risk factors for Common Mental Disorders among young people aged 10-20 years: A structured review of quantitative research. Health \& Place, 20, 81-90.

Diez Roux, A. V., \& Mair, C. (2010). Neighborhoods and health. Annals of the New York Academies of Science, 1186, 125-145.

Dobbie, W., \& Fryer, R. G. (2011). Are high-quality schools enough to increase achievement among the poor? Evidence from the Harlem Children's Zone. American Journal of Applied Economics, 3(3), 158-187.

Downey, L. (2006). Using geographic information systems to reconceptualize spatial relationships and ecological context. American Journal of Sociology, 112, 567-612.

Dupere, V., Leventhal, T., \& Vitaro, F. (2012). Neighborhood processes, self-efficacy, and adolescent mental health. Journal of Health and Social Behavior, 53(2), 183-198. 
Entwisle, D. R., Alexander, K. L., \& Olson, L S. (2005). First grade and educational attainment by age 22: A new story. American Journal of Sociology, 110(5), 1458-1502.

Flowerdew, R., Manley, D. J., \& Sabel, C. E. (2008). Neighbourhood effects on health: Does it matter where you draw the boundaries? Social Science \& Medicine, 66(6), 1241-1255.

Forsyth, A., Wall, M., Larson, N., Story, M., \& Neumark-Sztainer, D. (2012). Do adolescents who live or go to school near fast-food restaurants eat more frequently from fast-food restaurants? Health \& Place, 18(6), 1261-1269.

Galster, G. (2008). Quantifying the effect of neighbourhood on individuals: Challenges, alternative approaches, and promising directions. Schmollers Jahrbuch, 128(1), 7-48.

Jencks, C., \& Mayer, S. (1990). The Social Consequences of Growing Up In a Poor Neighborhood. In L. E. Lynn \& M. F. H. McGeary (Eds.), Inner-City Poverty In the United States (pp. 111-186). Washington, DC: National Academy Press.

Jones, M., \& Pebley, A. R. (2014). Redefining neighborhoods using common destinations: Social characteristics of activity spaces and home census tracts compared. Demography, $51,727-752$.

Kohen, D. E., Dahinten, V. S., Leventhal, T., \& McIntosh, C. N. (2008). Neighborhood disadvantage: Pathways of effects for young children. Child Development, 79(1), 156169.

Lareau, A. (2003). Unequal Childhoods: Class, Race, and Family Life. Berkley, CA: University of California Press. 
Leventhal, T., \& Brooks-Gunn, J. (2000). The neighborhoods they live in: The effects of neighborhood residence on child and adolescent outcomes. Psychological Bulletin, 126(2), 309-337.

Leventhal, T., \& Brooks-Gunn, J. (2003). Children and youth in neighborhood contexts. [Article]. Current Directions in Psychological Science, 12(1), 27-31.

Leventhal, T., Dupere, V., \& Brooks-Gunn, J. (2009). Neighborhood Influences on Adolescent Development. In R. M. Lerner \& L. Steinberg (Eds.), Handbook of Adolescent Psychology (3rd ed., Vol. 2, pp. 411-443). Hoboken, NJ: Wiley.

National Center for Education Statistics. (2006). Combined User's Manual for the ECLS-K FifthGrade Data Files and Electronic Codebook (NCES-2006-032 revised).

Northridge, M. E., Sclar, E. D., \& Biswas, P. (2003). Sorting out the connections between the built environment and health: A conceptual framework for navigating pathways and planning healthy cities. Journal of Urban Health, 80(4), 556-568.

Owens, A. (2010). Neighborhoods and schools as competing and reinforcing contexts for educational attainment. Sociology of Education, 83(4), 287-311.

Phan, T. (2015). Documentation for the School Attendance Boundary Survey: School Year 2013-2014 (NCES 2015-118). U.S. Department of Education. Washington, DC: National Center for Education Statistics.

Roeser, R. W., \& Eccles, J. S. (2014). Schooling and the Mental Health of Children and Adolescents in the United States. In M. Lewis \& K. D. Rudolph (Eds.), Handbook of Developmental Psychopathology (Third ed., pp. 163-184). New York, NY: Spring Science+Business Media. 
Root, E. (2012). Moving Neighborhoods and Health Research Forward: Using Geographic Methods to Examine the Role of Spatial Scale in Neighborhood Effects on Health. Annals of the Association of American Geographers, 102(5), 986-995.

Rosenbaum, P., \& Rubin, D. (1983). The central role of the propensity score in observational studies for causal effects. Biometrika, 70(1), 41-55.

Rubinowitz, L. S., \& Rosenbaum, J. E. (2000). Crossing the Class and Color Lines: From Public Housing to White Suburbs. Chicago, IL: University of Chicago Press.

Sampson, R., Morenoff, J., \& Gannon-Rowley, T. (2002). Assessing "neighborhood effects": Social processes and new directions in research. Annual Review of Sociology, 28, 443478.

Sastry, N., \& Pebley, A. R. (2010). Family and neighborhood sources of socioeconomic inequality in children's achievement. Demography, 47(3), 777-800.

Schwartz, H. (2010). Housing policy is school policy: Economically integrative housing promotes academic success in Montgomery County, MD. New York: The Century Foundation.

Sharkey, P., \& Faber, J. W. (2014). Where, when, why and for whom do residential contexts matter? Moving away from the dichotomous understanding of neighborhood effects. Annual Review of Sociology, 40, 559-579.

Singer, J. D., \& Willett, J. B. (2003). Applied longitudinal data analysis: Modeling change and event occurrence. New York, New York: Oxford University Press.

Spielman, S. E., Yoo, E., \& Linkletter, C. (2013). Neighborhood contexts, health, and behavior: Understanding the role of scale and residential sorting. Environment and Planning B: Planning and Design, 40, 489-506. 
StataCorp. (2011). Stata Statistical Software: Release 12. College Station, TX: StataCorp LP.

The College of William and Mary and the Minnesota Population Center. (2011). School Attendance Boundary Information System (SABINS). Minneapolis, MN: University of Minnesota.

Tobler, W. (1970). A computer movie simulating urban growth in the Detroit region. Economic Geography, 46, 234-240.

Villaneuva, K., Giles-Corti, B., Bulsara, M., McCormack, G. R., Timperio, A., Middleton, N., Beesley, B., \& Trapp, G. (2012). How far do children travel from their homes? Exploring children's activity spaces in their neighborhood. Health \& Place,18, 263-273.

Whipple, S. S., Evans, G. W., Barry, R. L., \& Maxwell, L. E. (2010). An ecological perspective on cumulative school and neighborhood risk factors related to achievement. Journal of Applied Developmental Psychology, 31(6), 422-427.

Zhang, Z., Christoffel, K. K., Mason, M., \& Liu, L. (2006). Identification of contrastive and comparable school neighborhoods for childhood obesity and physical activity research. International Journal of Health Geography, 5. 